\title{
Paracetamol-induced perforation of the esophagus in a patient with eosinophilic esophagitis
}

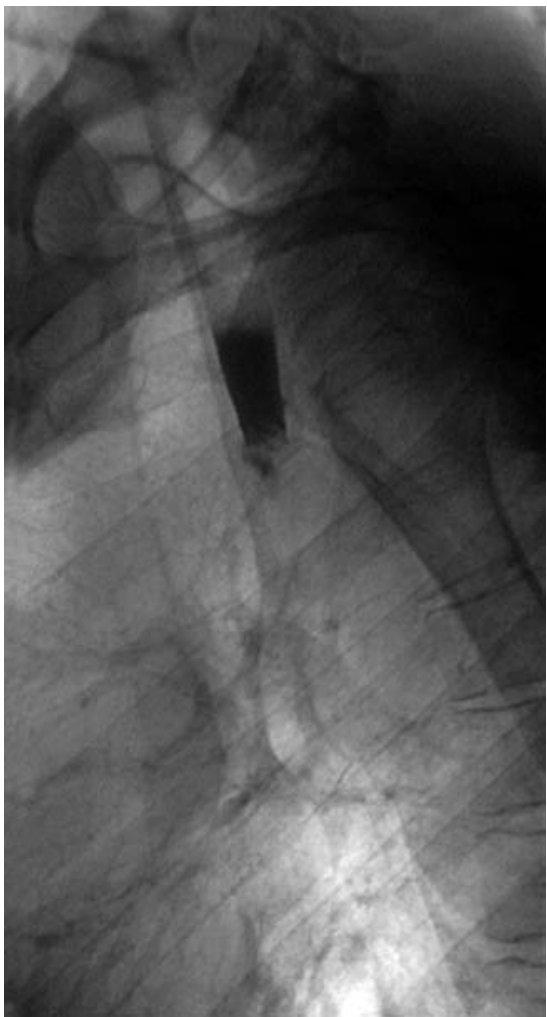

Fig. 1 Lateral esophagogram showing retention of contrast dye at the end of the proximal third of the esophagus consistent with foreign body retention.

A 41-year-old female patient admitted herself to the emergency room with dysphagia 18 hours after ingesting a tablet of paracetamol. She had suffered from multiple episodes of dysphagia before. As shown in Fig. 1, an upper gastrointestinal series radiograph taken after admission revealed obstruction of the proximal third of the esophagus. Emergency esophagoscopy revealed a dilated esophagus and the tablet at the end of the oral third of the esophagus ( $\bullet$ Fig. 2 a). After the tablet was gently mobilized, a membranous stricture and moderate hemorrhage became visible ( $\bullet$ Fig. $\mathbf{2}$ b). The patient subsequently developed chest pain, and computed tomography ( Fig. 3 ) showed mediastinal air consistent with perforation. The patient improved under conservative therapy and was discharged 5 days later. On day 40 she presented again with dysphagia and underwent manometry of the esophagus, which

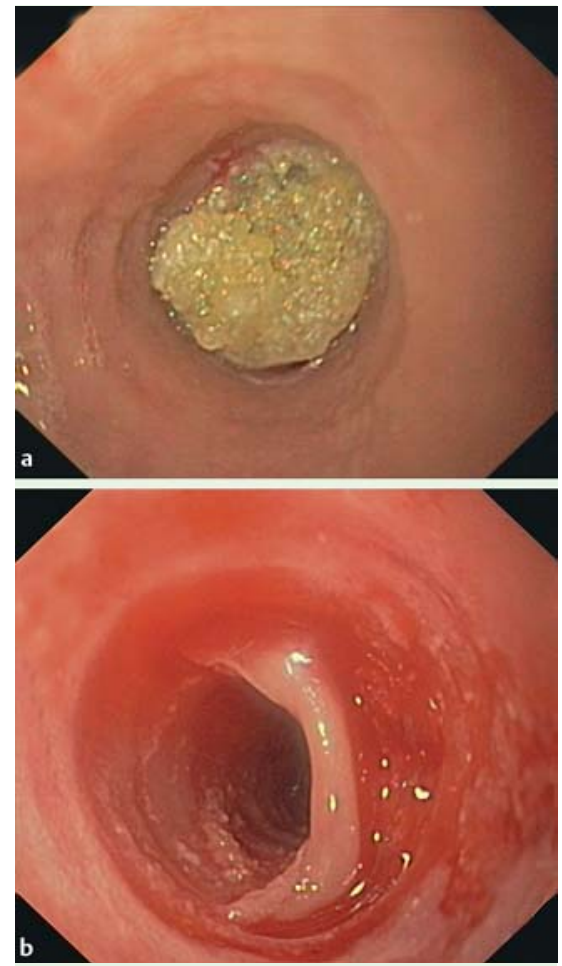

Fig. 2 Endoscopic aspect of the proximal esophagus prior to (a) and after (b) mobilization of the retained tablet. A soft tissue membrane becomes visible and the mucosal surface bleeds after contact with the endoscope.

showed no evidence of motility disorders. Esophagogastroduodenoscopy (EGD) revealed multiple, small, nodular lesions in the esophagus. Biopsies showed eosinophilic esophagitis ( Fig.4).

Relapsing dysphagia and bolus obstruction of the esophagus are frequent symptoms in patients with eosinophilic esophagitis [1]. The disease presents with esophageal rings and segmental stenosis of the esophagus [2], which might predispose to bolus impaction. Conversely, esophageal biopsies taken in patients presenting with bolus obstruction reveal eosinophilic esophagitis in up to $50 \%$ of cases [3]. This is the first case of combined pill-induced and eosinophilic esophagitis leading to perforation despite minimal endoscopic manipulation. In eosinophilic esophagitis, the rate of perforation following rigid esophagoscopy is as high as $20 \%$ [4], but it has also been described following flexible EGD without

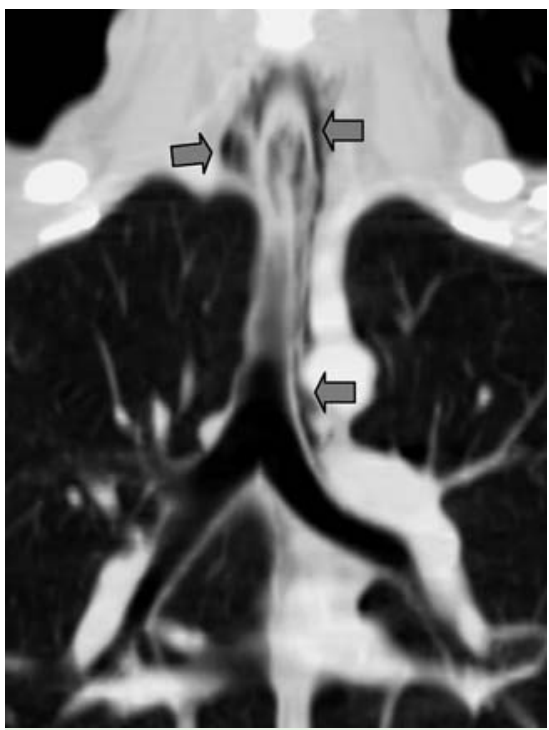

Fig. 3 Coronal reconstruction of thoracic computed tomography showing mediastinal air (arrows) in the cervical and paratracheal soft tissue.

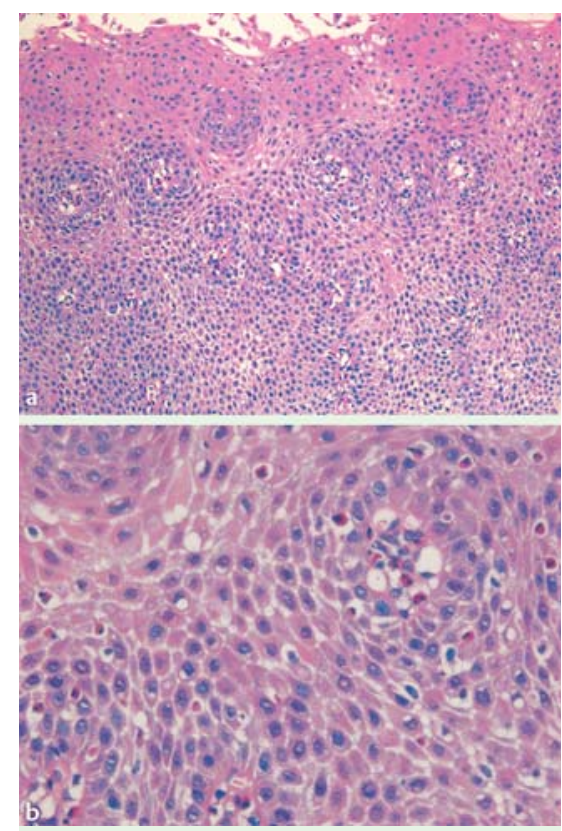

Fig. 4 Microphotographs of esophageal biopsy specimen. A typical aspect of eosinophilic esophagitis is shown, with more than 20 eosinophilic granulocytes per high-power field in the squamous epithelium of the esophageal mucosa, elongation of the papillae, dilatation of the intercellular spaces and basal cell hyperplasia. (Hematoxylin and eosin staining, original magnification: $\mathbf{a} \times 200 ; \mathbf{b} \times 600$.)

intervention [5]. Thus, eosinophilic esophagitis might increase the fragility of the esophageal wall, and great care is needed in the endoscopic treatment of patients presenting with a history of relapsing bolus obstruction.

Endoscopy_UCTN_Code_CCL_1AB_2AC_3AH 


\section{T. W. Spahn ${ }^{1,3}$, M. Vieth' ${ }^{2}$, M. K. Mueller ${ }^{1}$}

1 Department of General Internal Medicine and Gastroenterology, Marienhospital Osnabrueck, Academic Teaching Hospital of the University of Hannover Medical School, Osnabrueck, Germany

2 Institute of Pathology, Klinikum Bayreuth, Bayreuth, Germany

3 current address:

Marienkrankenhaus Schwerte, Goethestraße 19, 58239 Schwerte, Fax: +49-2304-109572

\section{References}

1 Müller S, Pühl S, Vieth $M$ et al. Analysis of symptoms and endoscopic findings in 117 patients with histological diagnoses of eosinophilic esophagitis. Endoscopy 2007; 39: 339-344

2 Dobbins JW, Sheahan DG, Behar J. Eosinophilic gastroenteritis with esophageal involvement. Gastroenterology 1977; 72: $1312-1316$

3 Kerlin $P$, Jones $D$, Remedios $M$ et al. Prevalence of eosinophilic esophagitis in adults with food bolus obstruction of the esophagus. J Clin Gastroenterol 2007; 41: 356-361

4 Straumann A, Bussmann C, Zuber M et al. Eosinophilic esophagitis: analysis of food impaction and perforation in 251 adolescent and adult patients. Clin Gastroenterol Hepatol 2008; 6: 598-600

5 Liguori G, Cortale M, Cimino F et al. Circumferential mucosal dissection and esophageal perforation in a patient with eosinophilic esophagitis. World J Gastroenterol 2008; 14: $803-804$

\section{Bibliography}

DOI $10.1055 / \mathrm{s}-2008-1077772$

Endoscopy 2010; 42: E31-E32

(c) Georg Thieme Verlag KG Stuttgart · New York . ISSN 0013-726X

\section{Corresponding author}

\section{T. W. Spahn, MD}

Marienhospital Osnabrueck

Bischofsstraße 1

D-49047 Osnabrueck

Germany

Fax: +49-541-3264656

heloto33@googlemail.com 